\title{
Labeling and Instructions for Use/Maintenance
}

National Cancer Institute

\section{Source}

National Cancer Institute. Labeling and Instructions for Use/Maintenance. NCI

Thesaurus. Code C92071.

Insufficient, inadequate, or incorrect information provided on a device's label or documentation regarding e.g. its intended use, directions for use, and characteristics of the device, including its maintenance. 